\title{
Solvent-Free Continuous Operations Using Small Footprint Reactors: A Key Approach for Process Intensification
}

\author{
Takashi Ouchi, ${ }^{\dagger}$ Robbie J. Mutton, ${ }^{\dagger}$ Victor Rojas, ${ }^{\ddagger}$ Daniel E. Fitzpatrick, ${ }^{\ddagger}$ David G. Cork, ${ }^{\dagger}$ \\ Claudio Battilocchio, ${ }^{*}+\frac{+}{*}$ and Steven V. Ley,
}

${ }^{\dagger}$ CMC Center, Chemical Development Laboratories, Takeda Pharmaceutical Company Limited, 17-85 Jusohonmachi 2-chome, Yodogawaku, Osaka 532-8686, Japan

${ }^{\ddagger}$ Innovative Technology Centre, Department of Chemistry, University of Cambridge, Lensfield Road, Cambridge CB2 1EW, United Kingdom

\section{Supporting Information}

ABSTRACT: A solvent-free approach to intensification of chemical processes is reported. This concept, in which reactions are carried out solvent-free, without the need for further downstream processing, was highlighted first with a full reduction of ethyl nicotinate to ethylpiperidine-3-carboxylate under continuous flow conditions. Using a small footprint reactor, a throughput of $26.4 \mathrm{~g} \mathrm{~h}^{-1}$ was achieved. Similarly, this approach was used for the telescoped two-step synthesis of 2 propyl phenol (productivity $120 \mathrm{~g} \mathrm{~h}^{-1}$ ) and 2-propyl cyclohexanone (productivity $30 \mathrm{~g} \mathrm{~h}^{-1}$ ), starting from the commercially available allyl phenol ether via Claisen rearrangement and subsequent reduction.

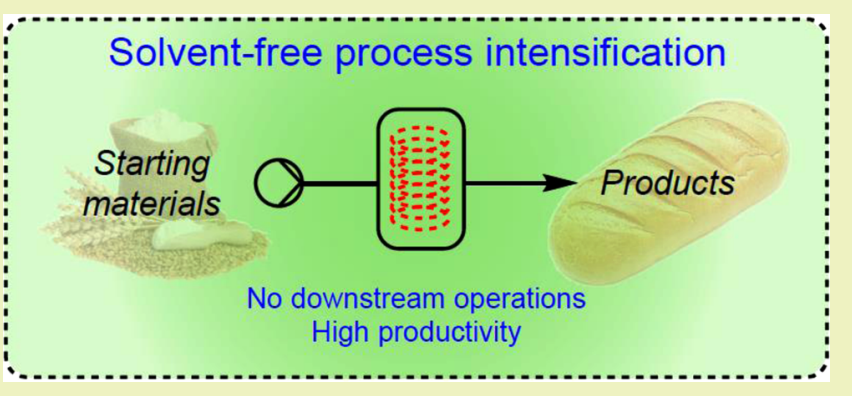

KEYWORDS: Claisen rearrangement, Flow chemistry, Heterogeneous hydrogenation, Process intensification, Solvent-free reactions

\section{INTRODUCTION}

Increasingly, there are demands placed on chemical manufacture, particularly arising from enhanced environmental awareness. The green agenda is evolving and correspondingly the industry is changing its approach in terms of planning and execution of chemical processes. ${ }^{1}$ By contrast, academia tends to be less constrained and a real step change is needed to respond positively to this new landscape.

In our own laboratory, we have attempted to bridge these different operating environments by focusing our attention on some aspects of chemical synthesis, specifically incorporating enabling technologies. ${ }^{2-14}$ In particular, the idea of using small footprint reactor platforms to perform intensive and repetitive tasks represents a very important area of development. This research indeed defines the starting point for intensifying chemical transformations, an essential strategy for future chemical manufacturing processes.

By definition, process intensification refers to a "strategy for making dramatic reductions in the size of a chemical plant". ${ }^{15}$ This strategy can be achieved first by using small footprint units for the production of large amounts of material. Second, process intensification can be attained through a reduction in the number of downstream operations, involving liquid separations, purifications, resolution, etc. The reduction of downstream operations has very important implications in reducing production timeframes, increasing productivity and reducing the overall process cost. For instance, if a process is set to reduce the quantities of solvents and water required for workup, a clear consequence is that there will be less waste to process and thus less impact with improved PMI (process mass intensity).

As a consequence, the ability to optimize processes and minimize downstream operations becomes an attractive strategy, especially if all downstream processing could be avoided.

Here we report the application of this specific approach in order to demonstrate its impact in a laboratory environment.

\section{FULL REDUCTION OF ETHYL NICOTINATE UNDER PROCESS INTENSIFICATION CONDITIONS}

We have recently reported on the initial intensification of a laboratory process (Scheme 1) to produce precursors used in the preparation of important pharmaceutical targets, e.g., Tiagabine (Figure 1). ${ }^{16}$ The methodology we employed focused on the partial and full reduction of ethyl nicotinate, using heterogeneous metal catalyzed hydrogenation, in order to achieve a specific high throughput of material.

To deliver this program, we made use of the HEL FlowCAT, a small footprint, robust trickle bed reactor that can manage high pressure and high temperature reactions. ${ }^{17}$

Received: February 9, 2016

Revised: March 10, 2016

Published: March 16, 2016 
Scheme 1. Previous Intensification Studies for the Partial (a) and Full (b) Reduction of Ethyl Nicotinate
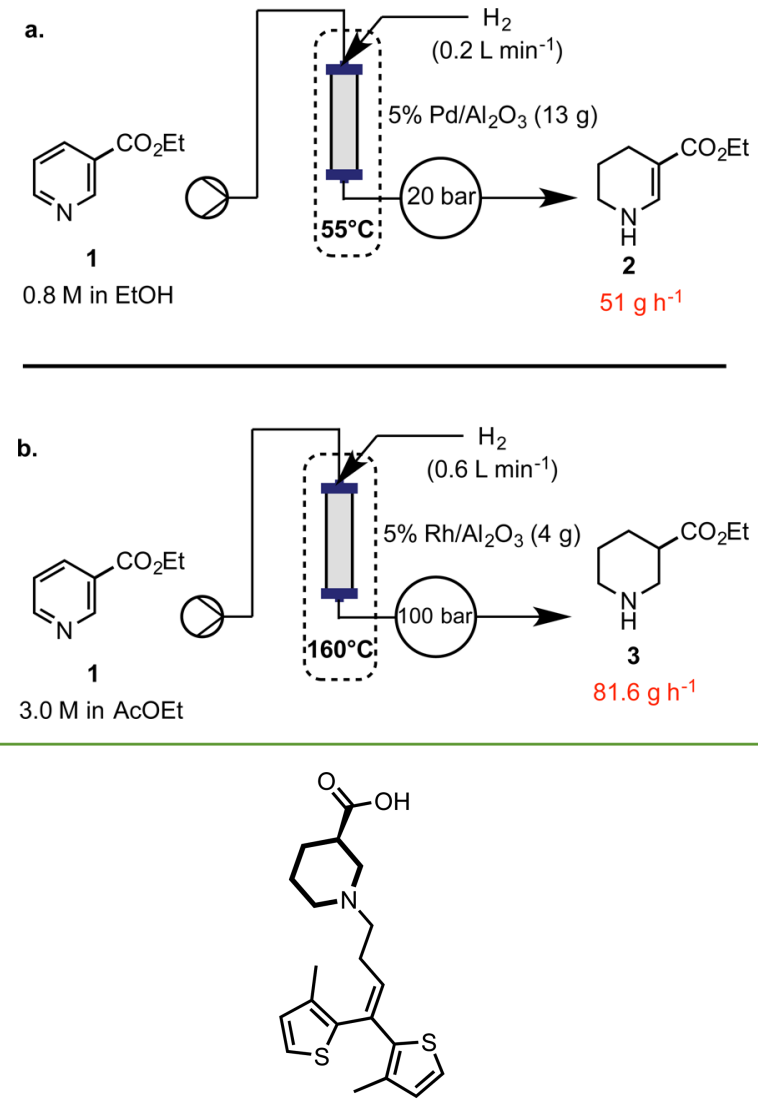

(R)-Tiagabine (Cephalon)

Figure 1. Tiagabine as an example of pharmaceuticals bearing the piperidine core.

To achieve our new goal of removing the need for any downstream processing following a reaction stage, we decided to challenge the system still further.

A first set of experiments was therefore conducted to verify the feasibility of a reaction where ethyl nicotinate (1) could be delivered without solvent (neat) into the reactor system where it would be fully reduced to the ethyl piperidin-3-carboxylic acid ester (3). We quickly noticed full reduction was possible and consequently decided to optimize the process. Following initial screening with various catalysts and conditions, it was found that running the reaction neat with a liquid feed of 0.5 $\mathrm{mL} \min ^{-1}$ and temperature of $160{ }^{\circ} \mathrm{C}$ resulted in $88 \%$ conversion, with a ratio $8: 1$ of fully/partially reduced material being observed, using $5 \% \mathrm{Rh} / \mathrm{Al}_{2} \mathrm{O}_{3}$ as catalyst. ${ }^{18,19}$

We continued these optimization efforts, identifying conditions that led ultimately to almost complete reduction of starting material to product 3 (around $1 \%$ of partially reduced compound was present in the reaction mixture). These conditions $\left(180{ }^{\circ} \mathrm{C}, 0.4 \mathrm{~mL} \mathrm{~min}{ }^{-1}\right.$, using $4 \mathrm{~g}$ of $\left.5 \% \mathrm{Rh} / \mathrm{Al}_{2} \mathrm{O}_{3}\right)$ allowed us to develop a robust protocol that could be applied in the laboratory on a multigram scale.

Indeed, with these conditions in hand, on running the reaction for $1 \mathrm{~h}$, it was possible to isolate $26 \mathrm{~g}$ of product with a purity of $\geq 96 \%$ (Scheme 2 ), with no downstream processing. We were very pleased to note that under these conditions the
Scheme 2. Ultimate Process Intensification for the Full Reduction of 1 to 3 under Solvent-Free Conditions

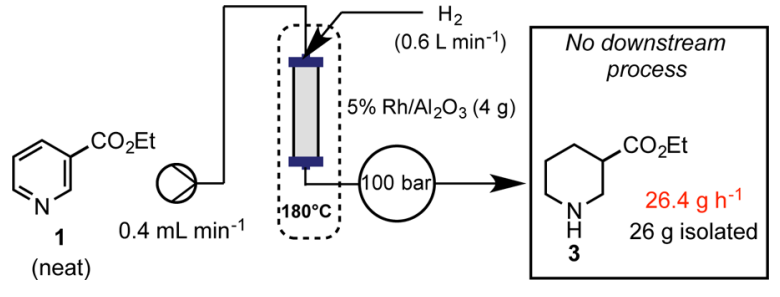

throughput of the process would equate to $639 \mathrm{~g} \mathrm{~d}^{-1}$, providing the catalyst remained productive. ${ }^{20}$

\section{TWO-STEP CONTINUOUS ULTIMATE INTENSIFIED PROCESSES}

One major benefit of continuous flow processing is the ability to telescope reaction sequences, whereby the output from one reactor is transferred directly into the inlet of the next step (usually passing through one or two downstream processing stages). Under intensification principles, the modus operandi would allow for significant cost savings, increased efficiencies and again reduced environmental impact for the transformations.

Although other groups have reported synthesis under solvent-free (neat) flow conditions, ${ }^{21,22}$ we felt there was a lack of general knowledge and literature regarding the use of small footprint platforms to perform these continuous solventfree operations, especially on a larger laboratory scale. Accordingly, we devised a sequence of steps under these telescoped flow conditions, to highlight opportunities of these methods.

Generation of 2-propyl phenol (6) and 2-propyl cyclohexanone (7) on a kilogram-scale were chosen as valuable targets commonly used in the flavor and fragrance industry (Scheme 3). ${ }^{23,24}$ The transformation process consisted of two

Scheme 3. Envisaged Strategy to Showcase a Two-Step Fully Intensified Protocol for the Synthesis of Target Compounds 6 and 7

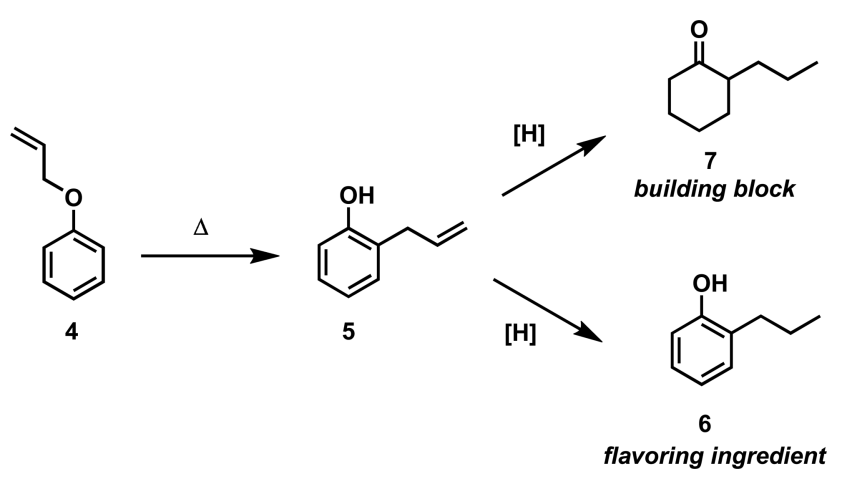

discrete reactions, namely a Claisen rearrangement followed by an hydrogenation step, starting from cheap and available feedstock material (allyl phenyl ether, 4).

To deliver the first step under intensified conditions, a new powerful, small footprint reactor was selected in order to process material at very high temperatures and pressures. ${ }^{25-27}$ The Phoenix reactor (ThalesNano) allows researchers to conduct reactions within underutilized chemical processing windows (Figure 2). ${ }^{28}$ The Phoenix reactor consists of two 


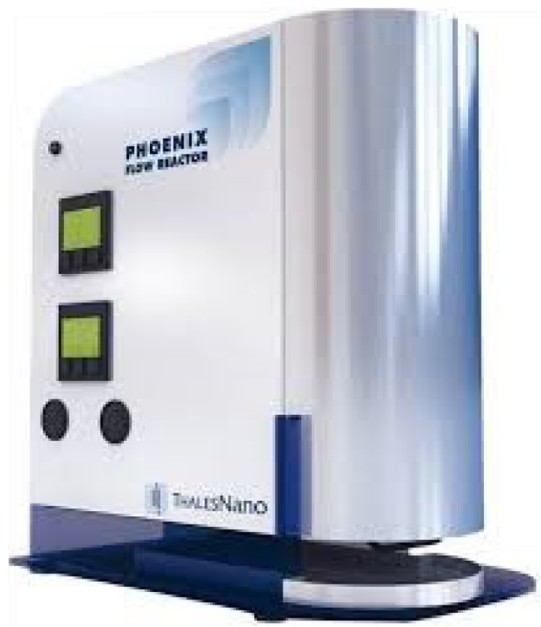

Figure 2. Phoenix Flow Reactor (copyright ThalesNano, reproduced with permission).

independently controlled, highly insulated heating elements, allowing for reactions to be performed between room temperature and $400{ }^{\circ} \mathrm{C}$. Within the central core of the heating elements, different reaction vessels of various size and function can be accommodated. 1 and $2 \mathrm{~mL}$ reaction vessels can either be used on their own or packed with solid reagents, whereas lengths of $1 / 16$ " stainless steel tubing can be coiled around a central column to give a range of known volume reactors that offer a high degree of thermal transfer allowing for faster flow rates and thus shorter residence times to be used. In addition, a separated compact back pressure regulator (BPR) unit facilitates reactions conducted between atmospheric pressure and 330 bar. The unit is able to communicate with external devices through RS232, enabling equipment parameters to be collected and recorded by control systems. ${ }^{29}$ Commands can also be received by the Phoenix to adjust set points, valve positions and power the device on and off. When operating at high temperatures and pressures, the ability to remotely monitor equipment status is extremely beneficial from a safety perspective.

A set of predetermined experiments quickly identified optimum conditions (see Supporting Information), affording an impressive $60 \mathrm{~g} \mathrm{~h}^{-1}$ with just $1 \mathrm{~min}$ residence time at $320^{\circ} \mathrm{C}$ and 100 bar of system pressure (Scheme 4$){ }^{30}$

Scheme 4. Intensified Claisen Rearrangement to Afford 5 using the Phoenix (ThalesNano) System
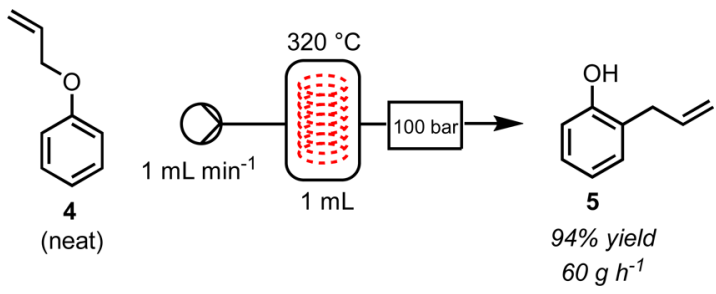

We were therefore satisfied with the level of intensification for this individual process. However, we decided to explore if we could further intensify the process by increasing the reactor capacity, with the hope that the system would respond linearly. To achieve this, the $1 \mathrm{~mL}$ reactor was substituted for an $8 \mathrm{~mL}$ reaction coil. Pleasingly, the linear increase of the liquid feed (from 1 to $8 \mathrm{~mL} \mathrm{~min}^{-1}$ ) afforded the same level of conversion and isolated yield for the product, giving an important $480 \mathrm{~g}$ $\mathrm{h}^{-1}$ throughput of material being processed, with $240 \mathrm{~g}$ of product being produced after just $30 \mathrm{~min}$ run.

Having quickly intensified the conditions for the Claisen step, we focused on the selective reduction of 5 to 6 . Again, our knowledge in the field of continuous heterogeneous hydrogenation allowed us to generate a table of relevant experiments (see Supporting Information), leading to suitable conditions for selective reduction. At a temperature of $80{ }^{\circ} \mathrm{C}$, liquid feed of 1 $\mathrm{mL} \min ^{-1}\left(\mathrm{H}_{2}\right.$ feed of $\left.0.4 \mathrm{~L} \mathrm{~min}^{-1}\right)$, and using $10 \% \mathrm{Pd} / \mathrm{C}^{18}$ as catalyst $(2 \mathrm{~g})$, phenol 5 was reduced to 6 with quantitative conversion and $98 \%$ yield (Scheme 5).

Scheme 5. Neat Reduction of 5 to 6 under Intensified Conditions

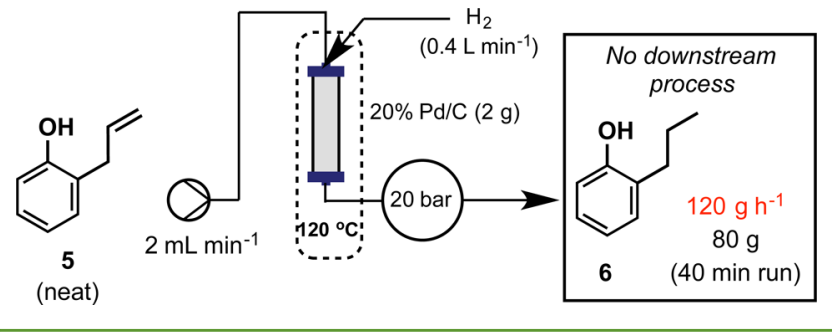

To intensify the reduction and increase the productivity of the process, we replaced $10 \% \mathrm{Pd} / \mathrm{C}$ with the higher loading $20 \% \mathrm{Pd} / \mathrm{C}^{18}$ Using this catalyst, we were able to push the throughput to $120 \mathrm{~g} \mathrm{~h}^{-1}\left(2 \mathrm{~mL} \mathrm{~min}^{-1}, 120{ }^{\circ} \mathrm{C}, 0.4 \mathrm{~L} \mathrm{~min}^{-1}\right)$, maintaining a very good level of efficiency $(\geq 94 \%$ product purity).

However, increasing the size of the column reactor (from 3 to $12 \mathrm{~mL}$ ) and the amount of catalyst did not result in a further appreciable scaleup. Nevertheless, we considered these conditions sufficient to demonstrate the concept of our studies.

To prove the concept of telescoping under neat and intensified conditions, we decided to run the experiments in a sequence, producing around $100 \mathrm{~g}$ of 6 in just $50 \mathrm{~min}$ (Scheme 6). In this case, we adapted the system in order to start collecting 5 while simultaneously processing it through the next step. This process performance produced 6 in $94 \%$ yield.

Our studies went further as we wanted to highlight the flexibility of the platform and the concept. Indeed, we aimed to identify the optimum conditions for the selective reduction of 5 to 7 (Scheme 7) and then telescope this stage with the previously mentioned Claisen step. The first set of reaction conditions gave important information that the solvent-free reaction to produce 7 was mainly dependent on temperature. To maintain a suitable throughput of material, $20 \% \mathrm{Pd} / \mathrm{C}^{18}$ is preferred as well as a temperature of $210{ }^{\circ} \mathrm{C}$. Under these conditions, compound 7 was obtained with a productivity of 30 $\mathrm{g} \mathrm{h}^{-1}$ (93\% yield).

Similarly to what we reported for the partial hydrogenation, we attempted the telescoped synthesis of 7 starting from 4 . Under these operating circumstances, we were able to produce several grams of product $(21.9 \mathrm{~g})$ reliably and with acceptable levels of purity ( $\geq 95 \%$, Scheme 7$)$.

\section{CONCLUSIONS}

Simple but powerful examples of process intensification under solvent-free conditions for continuous single and telescoped flow operations have been demonstrated. In both cases, it was possible to extend the capabilities of commercially available, 
Scheme 6. Telescoped Intensified Claisen Rearrangement and Reduction to 6

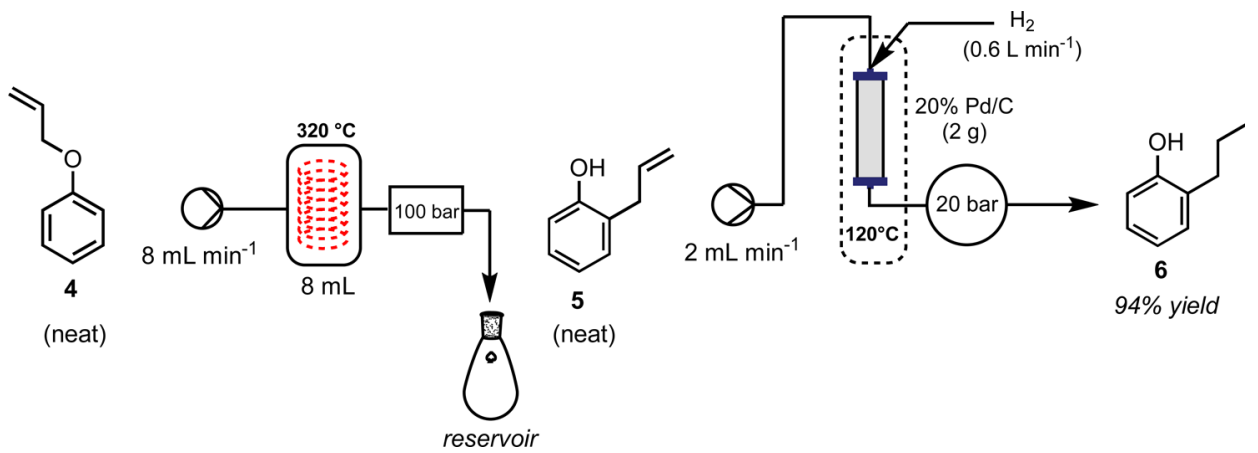

Scheme 7. Telescoped Intensified Claisen Rearrangement Followed by Full Reduction To Afford 7
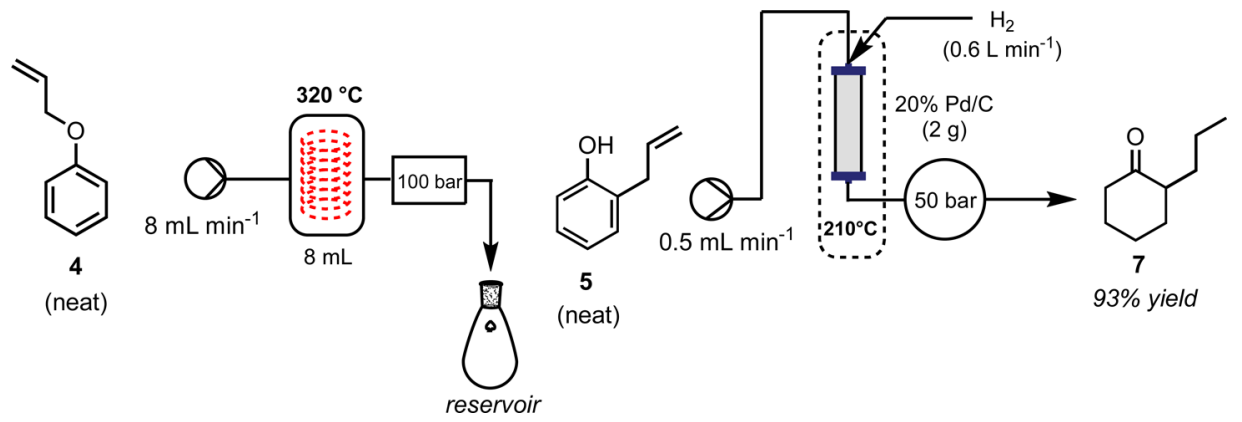

small footprint flow reactors. Such an approach in an appropriate environment could lead to significant cost savings and increases in efficiency. These examples of laboratory process intensification constitute possible benchmarks for further development in the field.

\section{ASSOCIATED CONTENT}

\section{S Supporting Information}

The Supporting Information is available free of charge on the ACS Publications website at DOI: 10.1021/acssuschemeng.6b00287.

Synthetic procedures and characterization of compounds (PDF).

\section{AUTHOR INFORMATION}

\section{Corresponding Authors}

*Dr. Claudio Battilocchio. Email: cb700@cam.ac.uk.

*Prof. Steven V. Ley. Email: svl1000@cam.ac.uk.

\section{Author Contributions}

The paper was written through contributions of all authors. All authors have given approval to the final version of the paper.

\section{Funding}

The EPSRC, grant codes EP/K009494/1, EP/M004120/1 and $\mathrm{EP} / \mathrm{K} 039520 / 1$.

\section{Notes}

The authors declare no competing financial interest.

\section{ACKNOWLEDGMENTS}

We thank Pfizer Worldwide Research \& Development (CB), the EPSRC (RJM, SVL, Grant Codes EP/K009494/1, EP/ M004120/1 and EP/K039520/1) and the Woolf Fisher Trust (DEF) for financial support. We thank Dr. Joel M. Hawkins (Pfizer Worldwide Research \& Development) for the very useful discussion of the project. We are grateful to Andrew
Coleman (HEL group) and Richard Jones (ThalesNano) for their support with the equipment. We are also grateful to Dr. Richard Turner and Dr. Megumi Nakano for providing assistance throughout the development of the project. We acknowledge ThalesNano for providing copyright permission for Figure 2. Additional data related to this publication is available at the University of Cambridge data repository at https://www.repository.cam.ac.uk/handle/1810/253708.

\section{REFERENCES}

(1) Green Chemistry in the Pharmaceutical Industry; Wiley-VCH: Weinheim, 2010.

(2) Battilocchio, C.; Feist, F.; Hafner, A.; Simon, M.; Tran, D. N.; Allwood, D. M.; Blakemore, D. C.; Ley, S. V. Iterative reactions of transient boronic acids enable sequential $\mathrm{C}-\mathrm{C}$ bond formation. Nat. Chem. 2016, DOI: $10.1038 /$ nchem.2439.

(3) Nagaki, A.; Imai, K.; Ishiuchi, S.; Yoshida, J. Reactions of difunctional electrophiles with functionalized aryllithium compounds: remarkable chemoselectivity by flash chemistry. Angew. Chem., Int. Ed. 2015, 54, 1914-1918.

(4) Newby, J. A.; Blaylock, D. W.; Witt, P. M.; Turner, R. M.; Heider, P. L.; Harji, B. H.; Browne, D. L.; Ley, S. V. Reconfiguration of a continuous flow platform for extended operation: application to a cryogenic fluorine-directed ortho-lithiation reaction. Org. Process Res. Dev. 2014, 18, 1221-1228.

(5) Battilocchio, C.; Baxendale, I. R.; Biava, M.; Kitching, M. O.; Ley, S. V. A flow-based synthesis of 2-aminoadamantane-2-carboxylic acid. Org. Process Res. Dev. 2012, 16, 798-810.

(6) Mascia, S.; Heider, P. L.; Zhang, H.; Lakerveld, R.; Benyahia, B.; Barton, P. I.; Braatz, R. D.; Cooney, C. L.; Evans, J. M. B.; Jamison, T. F.; Jensen, K. F.; Myerson, A. S.; Trout, B. L. End-to-end continuous manufacturing of pharmaceuticals: integrated synthesis, purification, and final dosage formation. Angew. Chem., Int. Ed. 2013, 52, 1235912363.

(7) Hartwig, J.; Ceylan, S.; Kupracz, L.; Coutable, L.; Kirschning, A. Heating under high-frequency inductive conditions: application to the continuous synthesis of the neurolepticum olanzapine (Zyprexa). Angew. Chem., Int. Ed. 2013, 52, 9813-9817. 
(8) Kundu, S.; Bhangale, A. S.; Wallace, W. E.; Flynn, K. M.; Guttman, C. M.; Gross, R. A.; Beers, K. L. Continuous flow enzymecatalyzed polymerization in a microreactor. J. Am. Chem. Soc. 2011, 133, 6006-6011.

(9) Ingham, R. I.; Battilocchio, C.; Hawkins, J. M.; Ley, S. V. Integration of enabling methods for the automated flow preparation of piperazine-2-carboxamide. Beilstein J. Org. Chem. 2014, 10, 641-652.

(10) Ley, S. V.; Fitzpatrick, D. E.; Ingham, R. J.; Myers, R. M. Organic synthesis: march of the machines. Angew. Chem., Int. Ed. 2015, 54, 3449-3464.

(11) Baumann, M.; Baxendale, I. R. The synthesis of active pharmaceutical ingredients (APIs) using continuous flow chemistry. Beilstein J. Org. Chem. 2015, 11, 1194-1219.

(12) Ley, S. V.; Fitzpatrick, D. E.; Myers, R. M.; Battilocchio, C.; Ingham, R. J. Machine-assisted organic synthesis. Angew. Chem., Int. Ed. 2015, 54, 10122-10136.

(13) Hessel, V.; Kralisch, D.; Kockmann, N.; Noël, T.; Wang, Q. Novel process windows for enabling, accelerating, and uplifting flow chemistry. ChemSusChem 2013, 6, 746-789.

(14) Pastre, J. C.; Browne, D. L.; Ley, S. V. Flow chemistry syntheses of natural products. Chem. Soc. Rev. 2013, 42, 8849-8869.

(15) Stankiewicz, A.; Moulijn, J. A. Process intensification. Ind. Eng. Chem. Res. 2002, 41, 1920-1924.

(16) Ouchi, T.; Battilocchio, C.; Hawkins, J. M.; Ley, S. V. Process intensification for the continuous flow hydrogenation of ethyl nicotinate. Org. Process Res. Dev. 2014, 18, 1560-1566.

(17) http://www.helgroup.com/reactor-systems/hydrogenationcatalysis/flowcat/ (accessed January 18, 2016).

(18) Catalyst was purchased from Johnson Matthey: http://www. matthey.com/johnson-matthey-catalysts.

(19) The reaction gave a specific ratio of $\mathbf{1 : 2 : 3}$ equating to 1.8:10.5:87.7.

(20) This is the case with a solution phase equivalent of this process.

(21) Snead, D. R.; Jamison, T. F. End-to-end continuous flow synthesis and purification of diphenhydramine hydrochloride featuring atom economy, in-line separation, and flow of molten ammonium salts. Chem. Sci. 2013, 4, 2822-2827.

(22) Sharma, Y.; Joshi, R. A.; Kulkarni, A. A. Continuous-Flow Nitration of $o$-Xylene: Effect of Nitrating Agent and Feasibility of Tubular Reactors for Scale-Up. Org. Process Res. Dev. 2015, 19, 11381147.

(23) http://www.ifraorg.org/en/ingredients\#.Vp1MGCqLSUk (accessed January 18, 2016).

(24) Method for the preparation of alpha-substituted organic carbonyl compounds. U.S. Patent US2773099A, December 4, 1956.

(25) Chaudhuri, R.; Hartwig, J.; Kupracz, L.; Kodanek, T.; Wegner, J.; Kirschning, A. Oxidations of Allylic and Benzylic Alcohols under Inductively-Heated Flow Conditions with Gold-Doped Superparamagnetic Nanostructured Particles as Catalyst and Oxygen as Oxidant. Adv. Synth. Catal. 2014, 356, 3530-3538.

(26) Sauks, J. M.; Mallik, D.; Lawryshyn, Y.; Bender, T.; Organ, M. G. A Continuous Flow Microwave Reactor for Conducting High Temperature and High Pressure Chemical Reactions. Org. Process Res. Dev. 2014, 18, 1310-1314.

(27) Sedelmeier, J.; Baxendale, I. R.; Ley, S. V. An Efficient and Transition Metal Free Protocol for the Transfer Hydrogenation of Ketones as a Continuous Flow Process. Green Chem. 2009, 11, 683685.

(28) http://thalesnano.com/phoenix-flow-reactor (accessed January $18,2016)$.

(29) Fitzpatrick, D. E.; Battilocchio, C.; Ley, S. V. A novel internetbased reaction monitoring, control and autonomous self-optimization platform for chemical synthesis. Org. Process Res. Dev. 2016, 20, 386394.

(30) For the Claisen rearrangement, our investigations have shown a decrease in conversion corresponding to a decrease of pressure; a similar observation was reported by the Hessel and coworkers: Kobayashi, H.; Driessen, B.; van Osch, D. J. G. P.; Talla, A.; Ookawara,
S.; Noël, T.; Hessel, V. The impact of novel process windows on the Claisen rearrangement. Tetrahedron 2013, 69, 2885-2890.

\section{NOTE ADDED AFTER ASAP PUBLICATION}

This article published March 18, 2016 with an incorrect value of the 2-propyl phenol productivity. The correct value published March 21, 2016. 